\title{
ABSORÇÃO DE GASES DA QUEIMA DE COMBUSTÍVEIS FÓSSEIS EM TORRE DE RECHEIO ESTRUTURADO UTILIZANDO LAMA VERMELHA
}

\author{
I. F. SANTOS ${ }^{1}$, L. C. A. VENANCIO ${ }^{1}$, G. P. C. CÉSAR ${ }^{2}$, J. A. S. SOUZA ${ }^{1}$ e C.A.G de SOUZA ${ }^{3}$. \\ ${ }^{1}$ Universidade Federal do Pará, Programa de Pós Graduação em Engenharia de Recursos Naturais \\ da Amazônia \\ ${ }^{2}$ Universidade Federal do Pará, Faculdade de Engenharia Química \\ ${ }^{3}$ Universidade Federal do Pará, Programa de Pós Graduação em Engenharia Química \\ E-mail para contato: iaraferreira.eq@gmail.com
}

\begin{abstract}
RESUMO - A produção de alumina pelo processo Bayer produz de 1 a 3 toneladas de lama vermelha por tonelada de alumínio. Esse resíduo possui em sua composição variados teores de $\mathrm{NaOH}$, e portanto, elevado $\mathrm{pH}$. Esse trabalho busca avaliar o processo de absorção de gases provenientes de uma caldeira por uma suspensão de lama vermelha a $27 \%$, em torre spray e em torre de recheio estruturado, processo que ocorre através de reações de carbonatação. Foram realizados experimentos variando o tipo de torre de absorção, temperatura e teor de sólidos na fase líquida para avaliar a redução do teor dos gases na saída das torres e a variação do $\mathrm{pH}$ ao longo do tempo de operação, para isso foram realizadas medições dos teores de gases na entrada e na saída das torres, medições de temperatura e pH. Os resultados obtidos após os experimentos foram satisfatórios, e concluiu-se que o processo realizado nas torres de absorção é eficiente para a redução do $\mathrm{pH}$ da lama vermelha e do teor de $\mathrm{CO}_{2}$ liberado para a atmosfera.
\end{abstract}

\section{INTRODUÇÃO}

Entre as atividades que contribuem para o aumento das emissões de $\mathrm{CO}_{2}$ para a atmosfera, dá-se ênfase na produção de energia através da queima de combustíveis fósseis. Segundo Venancio (2010), em refinaria de alumina, utilizando o processo Bayer, localizada em BarcarenaPará (Hydro-ALUNORTE) utiliza-se um óleo com alto teor de carbono como combustível na caldeira de produção de vapor e no calcinador de alumina, gerando grande quantidade de gases de combustão com elevado teor de carbono. Outro problema ambiental referente ao processo Bayer é a grande quantidade de resíduo formado a partir do processamento da bauxita, denominado "lama vermelha", que apesar de não ser considerado um resíduo tóxico apresenta um pH muito elevado, o que pode acarretar uma série de problemas ambientais caso não seja armazenado ou descartado de maneira correta. No presente trabalho é estudada a viabilidade de utilizar a lama vermelha e os gases de combustão em um processo de absorção, buscando-se reduzir os impactos ambientais causados por esses dois rejeitos provenientes da indústria de produção de alumina.

Em diversos estudos encontrados na literatura, as reações de carbonatação que ocorrem entre compostos alcalinos e o $\mathrm{CO}_{2}$, quando em contato, são citadas e exemplificadas, na forma de equações químicas. As reações de carbonatação são exotérmicas e apresentam uma forma de captura de carbono segura na forma de carbonatos (Bobicki et al., 2012). Jones et al. (2006), Sahu 
et al. (2010a), Venancio (2010), Santos (2013) entre outros, já abordaram o processo de absorção de $\mathrm{CO}_{2}$ pela lama vermelha, obtendo bons resultados. Segundo Jones et al. (2006), a carbonatação para neutralizar soluções cáusticas de aluminatos ocorre da seguinte forma: o $\mathrm{CO}_{2}$ reage com o íon hidroxila, presente nas soluções cáusticas, produzindo o íon bicarbonato, que por sua vez se dissocia em íons hídron $\left(\mathrm{H}^{+}\right)$e carbonato. A reação final indica que existe um equilíbrio entre as taxas de bicarbonato/carbonato, porém, de acordo com o $\mathrm{pH}$ de equilíbrio da lama após o processo de carbonatação, o equilíbrio é fortemente deslocado em favor do íon bicarbonato. Portanto, o íon bicarbonato pode ser visto como o armazenador de dióxido de carbono que participa das reações à medida que ocorre a redução do pH enquanto ele é consumido (Jones et al., 2006). Ainda segundo Jones et al. (2006), o aluminato presente na solução pode precipitar como hidróxido de alumínio ou reagir com o dióxido de carbono na solução e formar dawsonita. Depois da carbonatação, o licor é empobrecido de alumínio e hidróxido e se torna predominantemente uma solução de bicarbonato. O trialuminato de cálcio (TCA) é o componente alcalino predominante do resíduo da lama vermelha, e durante a carbonatação o TCA se dissolve para se combinar com o $\mathrm{CO}_{2}$ e se precipita como calcita e hidróxido de alumínio.

Segundo Bonefant et al. (2008), o processo de carbonatação consiste em várias reações de $\mathrm{CO}_{2}$ com compostos alcalinos presentes na fase líquida, para formar espécies de carbonato. De acordo com Dilmore et al. (2008), a dissolução de $\mathrm{CO}_{2}$ em água, resulta na formação de ácido carbônico que se dissocia formando os íons $\mathrm{HCO}^{3-}$ e $\mathrm{CO}_{3}{ }^{2-}$, liberando assim $\mathrm{H}^{+}$para o fluido, dessa forma, ocorre a redução do $\mathrm{pH}$ da solução. A redução do $\mathrm{pH}$ é ainda intensificada devido à precipitação dos íons presentes na solução na forma de carbonatos. A carbonatação é um processo seguro e barato, visto que os produtos formados a partir das reações químicas que ocorrem são estáveis e pode ser uma solução vantajosa para os problemas enfrentados com o armazenamento de lama vermelha e com as milhares de toneladas de $\mathrm{CO}_{2}$ que são emitidas anualmente pelas indústrias durante a produção de alumina. Além disso, a lama vermelha carbonatada apresenta uma série de benefícios associados ao seu uso.

\section{OBJETIVOS}

Avaliar o processo de absorção de $\mathrm{CO}_{2}$ por uma suspensão de lama vermelha em uma planta piloto, objetivando capturar e reduzir o teor de dióxido de carbono nos gases de combustão provenientes da queima de combustíveis fósseis, bem como modificar algumas das propriedades da lama vermelha que impedem o descarte no meio ambiente. Avaliar a eficiência de torres de absorção distintas de acordo com variação de temperatura, concentração da suspensão e características estruturais. Observar a taxa de variação do pH da suspensão durante o experimento.

\section{MATERIAIS E MÉTODOS}

\subsection{Materiais}

A lama vermelha (LV) utilizada para o preparo da suspensão que passa pelas torres de absorção nos experimentos realizados foi fornecida pela empresa Hydro-ALUNORTE (BarcarenaPará). Para o preparo da suspensão, a lama vermelha não passou por nenhum tratamento prévio, com o objetivo de avaliar se a suspensão preparada com o resíduo, a ser usada no processo de absorção, é eficiente utilizando o resíduo com as propriedades que o mesmo possui no final da etapa vermelha do processo Bayer, tornando o processo mais prático e econômico. Os gases de 
combustão utilizados nos experimentos são provenientes de uma caldeira que utiliza óleo diesel como combustível. Dentre os gases, dá-se destaque ao $\mathrm{CO}_{2}$, pois a partir dos valores de entrada e saída desse gás será possível avaliar o sequestro de carbono ocorrido no processo.

Equipamentos: A planta piloto existente no laboratório de engenharia química (Universidade Federal do Pará) para estudo do sequestro de carbono e neutralização do resíduo do processo Bayer contém três torres de absorção: Uma torre de aspersão (torre spray) e duas torres de recheio, sendo uma torre com recheios randômicos e uma contendo recheios estruturados. Neste trabalho fez-se um estudo da absorção de $\mathrm{CO}_{2}$ e redução de $\mathrm{pH}$ da suspensão de $\mathrm{LV}$ em apenas dois desses três reatores, a torre de aspersão e a torre de recheios estruturados, mostradas na Figura 1, juntamente com o recheio estruturado utilizado para o preenchimento da torre de absorção.

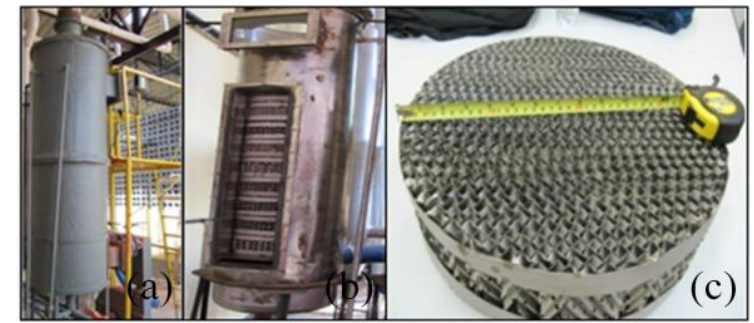

Figura 1 - Torre spray (TS) (a), torre de recheios estruturados (TRE) (b) e recheio estruturado (c).

\subsection{Metodologia Experimental}

Para o preparo da suspensão foram adicionados 80 litros de água no reservatório de fibrocimento com capacidade de 500 litros, em seguida, adicionou-se LV sem tratamento prévio, te obter-se a concentração de $1207 \mathrm{~g} / \mathrm{dm}^{3}$ aproximadamente, essa concentração foi medida com ajuda de uma proveta de $1000 \mathrm{~mL}$. Foi realizado um ajuste nas válvulas da planta piloto para que fosse possível a circulação da suspensão pela caixa de peneiramento, com o objetivo de retirar impurezas maiores, como por exemplo, pedras ou pedaços de lama endurecidos, para que não houvesse interferência durante a passagem da suspensão pelos reatores e nem entupimento dos bicos da torre spray. Esse valor de concentração consiste em $27 \%$ em peso de lama vermelha na suspensão, foi determinado por Venancio (2010) como o maior teor de sólido possível sem que ocorra entupimento dos bicos de aspersão.

Suspensão com defloculante: Para evitar que os sólidos da suspensão floculassem e dessa forma reduzir a quantidade de sólidos que entram em contato com a fase gasosa no reator, foi necessário utilizar 0,16 gramas de hexametafosfato de sódio como defloculante. Esse valor foi determinado por estudos feitos por Venancio (2010).

Suspensão com floculante: Alguns experimentos foram realizados com uma quantidade bem reduzida de sólidos presentes na suspensão para determinar a capacidade de sequestro de $\mathrm{CO}_{2} \mathrm{e}$ redução do $\mathrm{pH}$ da lama vermelha para processos que utilizassem apenas o sobrenadante da suspensão. Para isso foi utilizada 0,01 grama de sulfato de alumínio como floculante. Esse valor foi determinado a partir de um experimento de decantação, no qual determinou-se qual a melhor concentração de sulfato de alumínio por litro de suspensão. Após 24 horas do preparo da suspensão com o floculante foram realizados os experimentos. Para captar apenas o sobrenadante, foi necessário trabalhar com um captador operando em baixa turbulência que ficava imerso na parte aquosa da suspensão do resíduo, de acordo com a Figura 2 a. 
Elevação da temperatura: Para elevar a temperatura da suspensão foi necessário inserir resistências no tanque para que entrassem em contato com a suspensão. Após certo tempo de contato foi determinada a temperatura inicial de equilíbrio e então dado início ao processo de absorção. A Figura $2 \mathrm{~b}$ mostra o conjunto de resistência utilizado para o aquecimento.

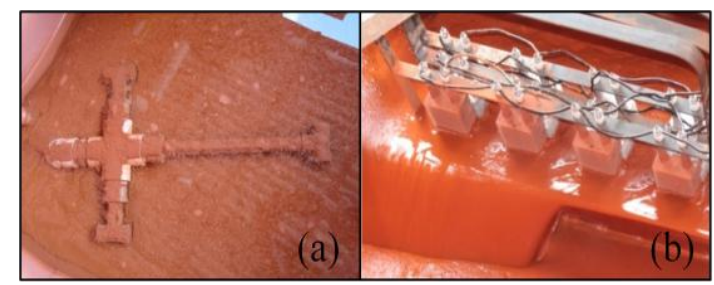

Figura 2 - Captador de sobrenadante (a) e conjunto de resistências (b).

Procedimento experimental: A fornalha da caldeira produz gases provenientes da queima de óleo combustível, os gases a serem utilizados no experimento passam por um trocador de calor para redução da temperatura. Após a redução de temperatura os gases são transportados para o manifold através do ventilador, e então levados até a torre de absorção selecionada, logo após passar pela torre o gás é então liberado pela chaminé. As medições das concentrações dos gases foram realizadas com a ajuda de dois analisadores de gases, que determinavam os teores de $\mathrm{CO}_{2}$, $\mathrm{O}_{2}, \mathrm{NO}_{\mathrm{X}}$ e $\mathrm{SO}_{2}$ na corrente gasosa, e realizadas nos pontos de coleta de dados 1 e 2, conforme Figura 3. Paralelamente, ao trajeto realizado pelos gases de combustão, a fase líquida (suspensão de LV ou sobrenadante) é bombeada, pela bomba centrífuga, até a torre de absorção e após a saída da torre é levada de volta ao reservatório, para que ocorra a recirculação da fase líquida, a medição do $\mathrm{pH}$ da suspensão foi realizada através de medidor de $\mathrm{pH}$, foi realizada antes do início bombeamento até o topo do reator e no ponto de coleta de dados 3 durante a realização do experimento. Para evitar a corrosão, o acúmulo de sólidos nos reatores (o que prejudicaria o processo de absorção devido à redução da área de troca térmica) e, no caso da torre spray, o entupimentos dos bicos aspersores, é necessário efetuar a lavagem das torres de absorção ao final de cada experimento com a ajuda da bomba centrífuga, através do ajuste de válvulas.

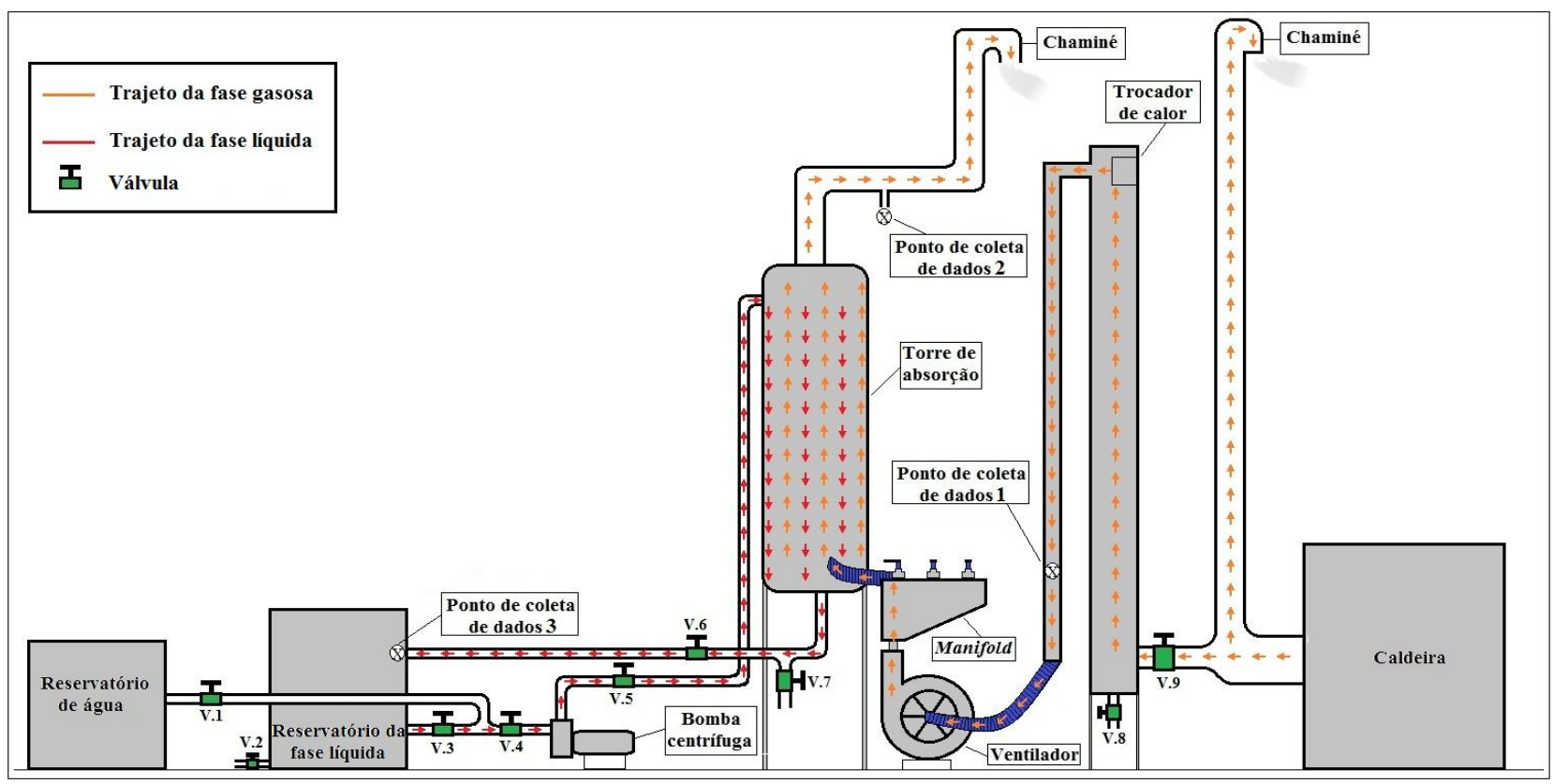

Figura 3 - Desenho esquemático da planta piloto utilizada no processo. 
Tratamento dos dados experimentais: Após a aquisição dos dados experimentais, obtiveramse os valores referentes à porcentagem de $\mathrm{CO}_{2}$ absorvido durante o experimento, a massa de $\mathrm{CO}_{2}$ absorvida a cada 100 gramas de LV presente na suspensão e a massa de $\mathrm{CO}_{2}$ absorvida no período de um ano, caso o processo ocorresse de maneira contínua, utilizando as Equações 1, 2 e 3, respectivamente.

$$
\begin{aligned}
& \% \mathrm{CO}_{2}(a b s)=\frac{\left(M_{t o t} \cdot \mathrm{CO}_{2}(a b s) \cdot 100 \%\right)}{M_{t o t} \cdot \mathrm{CO}_{2}(e)} \\
& M_{\mathrm{CO}_{2}(a b s) / 100 \mathrm{gdeLV}}=\frac{\left(M_{t o t} \cdot \mathrm{CO}_{2}(a b s) \cdot 100 \mathrm{~g}\right)}{33,5 \mathrm{~kg}} \\
& M_{\text {tot. } \mathrm{CO}_{2}(\text { abs }) \text { ano }}=\frac{\left(M_{\text {tot } . \mathrm{CO}_{2}(a b s)} \cdot 525600 \mathrm{~min}\right)}{t_{\text {exp }}}
\end{aligned}
$$

Onde:

$M_{\text {tot. } \mathrm{CO}_{2}(e)}$ : massa total de $\mathrm{CO}_{2}$ que entra no reator ao longo do experimento;

$M_{\text {tot. } \mathrm{CO}_{2}(\mathrm{abs})}$ : massa total de $\mathrm{CO}_{2}$ absorvida ao final do experimento;

$t_{\text {exp }}:$ tempo de duração do experimento.

De acordo com a concentração e o volume aproximado da suspensão, para cada experimento foi encontrado o valor médio de massa de LV existente na suspensão igual a $33,5 \mathrm{~kg}$, valor utilizado para cálculo da quantidade de $\mathrm{CO}_{2}$ absorvida para cada $100 \mathrm{~g}$ de LV de acordo. Para o cálculo da massa de $\mathrm{CO}_{2}$ que pode ser absorvido anualmente, foi utilizado o valor de 525600 minutos, referente à quantidade de minutos existentes em um ano com 365 dias.

\section{RESULTADOS E DISCUSSÃO}

Os experimentos (EXP) foram realizados de acordo com as informações contidas na Tabela 1, quanto ao tipo de reator utilizado (TS ou TRE), concentração de sólidos na fase líquida (suspensão ou sobrenadante) e temperatura inicial da fase líquida ( $\mathrm{T}_{\text {faselíquida }}$ ) (temperatura ambiente $\left(\mathrm{T}_{\mathrm{amb}}\right)$ ou temperatura alta $\left.\left(\mathrm{T}_{\mathrm{alta}}\right)\right)$.

Tabela 1 - Condições operacionais para realização dos experimentos.

\begin{tabular}{ccccccc}
\hline \multirow{2}{*}{ EXP } & \multicolumn{2}{c}{ Reator } & \multicolumn{2}{c}{ Fase líquida } & \multicolumn{2}{c}{$\mathrm{T}_{\text {fase líquida }}$} \\
\cline { 2 - 7 } & $\mathrm{TS}$ & $\mathrm{TRE}$ & Susp. & Sobr. & $\mathrm{T}_{\text {amb. }}$ & $\mathrm{T}_{\text {alta }}$ \\
\hline 01 & $\mathrm{X}$ & - & $\mathrm{X}$ & - & $\mathrm{X}$ & - \\
02 & $\mathrm{X}$ & - & - & $\mathrm{X}$ & $\mathrm{X}$ & - \\
03 & $\mathrm{X}$ & - & $\mathrm{X}$ & - & - & $\mathrm{X}$ \\
04 & - & $\mathrm{X}$ & - & $\mathrm{X}$ & - & $\mathrm{X}$ \\
05 & $\mathrm{X}$ & - & $\mathrm{X}$ & - & $\mathrm{X}$ & - \\
06 & $\mathrm{X}$ & - & - & $\mathrm{X}$ & - & $\mathrm{X}$ \\
07 & - & $\mathrm{X}$ & $\mathrm{X}$ & - & $\mathrm{X}$ & - \\
\hline
\end{tabular}

Os resultados obtidos para cada experimento foram comparados para avaliar a influência de 
cada fator para a eficiência da absorção, e as características consideradas mais importantes para avaliar o desempenho de cada experimento foram: variação do $\mathrm{pH}$ durante o experimento; porcentagem de $\mathrm{CO}_{2}$ absorvida; massa de $\mathrm{CO}_{2}$ absorvida a cada 100 gramas de LV da suspensão; massa de $\mathrm{CO}_{2}$ absorvida em um ano caso o processo se dê de forma contínua. Os experimentos tiveram duração de 80 minutos, e foram coletados os dados de temperatura inicial, $\mathrm{pH}$ da fase líquida durante o experimento e vazões das fases gasosa e líquida $\left(\mathrm{Q}_{\text {gás }} \mathrm{e} \mathrm{Q}_{\text {líquido }}\right)$ presentes na Tabela 2.

Tabela 2 - Dados experimentais de temperatura inicial e $\mathrm{pH}$ inicial e final.

\begin{tabular}{rrrrrr}
\hline EXP & $\mathrm{T}_{\text {inicial }}\left({ }^{\circ} \mathrm{C}\right)$ & $\mathrm{pH}_{\text {inicial }}$ & $\mathrm{pH}_{\text {final }}$ & $\mathrm{Q}_{\text {gás }}\left(\mathrm{m}^{3} / \mathrm{min}\right)$ & $\mathrm{Q}_{\text {líquido }}\left(\mathrm{m}^{3} / \mathrm{min}\right)$ \\
\hline 01 & 27 & 12,6 & 9,6 & 1,5558 & 0,0618 \\
02 & 27 & 12,8 & 8,3 & 1,3536 & 0,0660 \\
03 & 48 & 12,3 & 9,0 & 1,6440 & 0,0756 \\
04 & 46 & 12,5 & 8,3 & 1,3530 & 0,0528 \\
05 & 27 & 12,3 & 9,4 & 1,3680 & 0,0444 \\
06 & 40 & 12,8 & 8,7 & 1,2186 & 0,0168 \\
07 & 27 & 12,9 & 8,1 & 0,9720 & 0,0582 \\
\hline
\end{tabular}

A seguir, na Figura 4, é mostrada a curva de variação de $\mathrm{pH}$ ao longo do procedimento experimental.

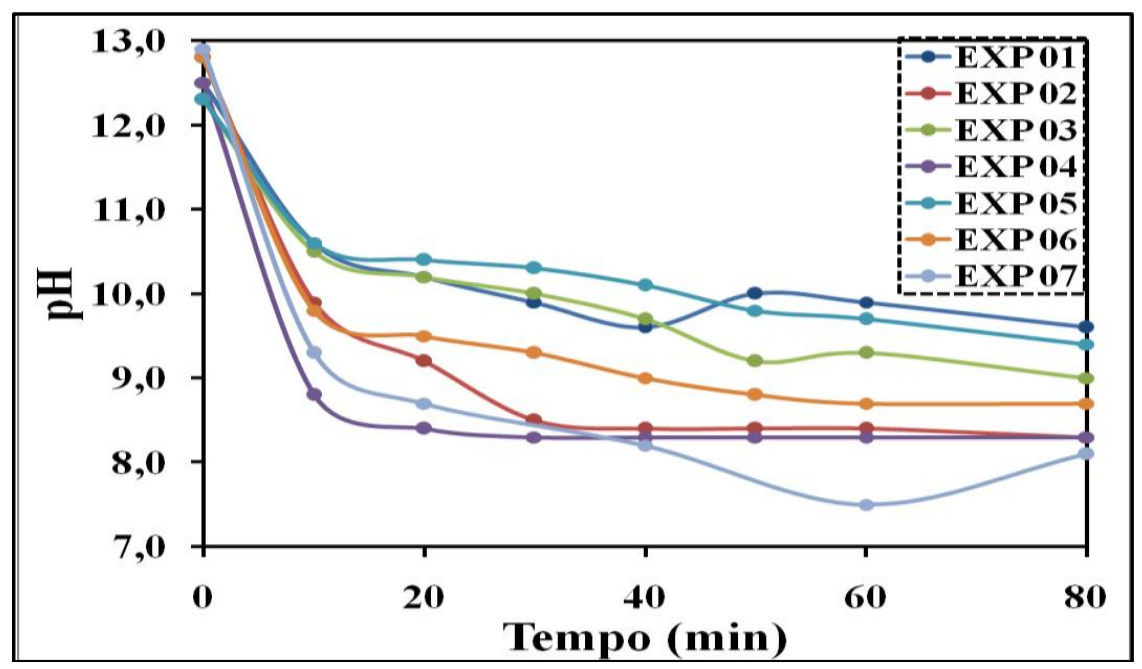

Figura 4 - Variação do pH em função do tempo.

Na Figura 6, observa-se que alguns experimentos apresentam uma menor inclinação inicial na curva de variação do pH que os demais, os experimentos 02, 04, 06 e 07 apresentam melhores resultados de redução de $\mathrm{pH}$ que os demais, e uma diminuição mais acentuada no valor do $\mathrm{pH}$, logo nos 20 primeiros minutos. Os experimentos 04 e 07 foram realizados em torre de recheio estruturado, que apresenta maior área de contato entre as fases, portanto, mais rapidamente se dá o processo de absorção de $\mathrm{CO}_{2}$ pela lama vermelha, e com isso uma maior redução inicial no valor do $\mathrm{pH}$ quando comparada aos experimentos 01, 03 e 05, realizados em torre spray, com menor área de contato entre as fases. Os experimentos 02 e 06 foram realizados em torre spray, porém, observa-se que há uma redução inicial acentuada no valor do $\mathrm{pH}$, nesse caso, fez-se uso do sobrenadante, e este reduz mais rapidamente o $\mathrm{pH}$ do que a suspensão, mostrando que a alcalinidade da lama vermelha se deve em sua maioria à parte sólida, e a alcalinidade da fase 
líquida é rapidamente reduzida. É possível notar em algumas curvas um aumento no valor do pH, durante o procedimento experimental, isso ocorre devido à medição de pH ser realizada logo após a mistura da suspensão ser feita, acontece que, em alguns instantes do experimento era necessário realizar uma mistura na suspensão presente no reservatório de fibrocimento, pois apesar do uso de defloculante ainda ocorre uma leve sedimentação da suspensão, portanto, ao misturá-la há um aumento na quantidade de sólidos presentes na suspensão que entrará em contato com a fase gasosa, e como a fase sólida da suspensão é responsável por grande parte da alcalinidade da lama vermelha isso implica no aumento do $\mathrm{pH}$.

Os experimentos são comparados entre si quanto à variação do $\mathrm{pH}$, porcentagem de $\mathrm{CO}_{2}$ absorvido e valores mássicos de absorção, para que seja possível avaliar a eficiência da absorção de acordo com a variação de algumas propriedades do processo, esses valores comparativos são mostrados na Tabela 3.

Tabela 3 - Valores de redução do pH, absorção de $\mathrm{CO}_{2}$ e valores mássicos de absorção.

\begin{tabular}{lllll}
\hline EXP & $\Delta \mathrm{pH}$ & $\begin{array}{l}\text { Absorção } \\
\text { de } \mathrm{CO}_{2}(\%)\end{array}$ & $\begin{array}{l}\text { Massa de } \mathrm{CO}_{2} \text { absorvida } \\
(\mathrm{g}) / 100 \mathrm{~g} \mathrm{de} \mathrm{LV}\end{array}$ & $\begin{array}{l}\text { Massa de } \mathrm{CO}_{2} \text { absorvida } \\
\text { anualmente }(\mathrm{kg})\end{array}$ \\
\hline 01 & 3,0 & 6,25 & 2,4673 & 5430,45 \\
02 & 4,5 & 9,26 & 3,8170 & 8401,04 \\
03 & 3,3 & 11,55 & 5,5571 & 12230,84 \\
04 & 4,2 & 4,35 & 1,5894 & 3498,21 \\
05 & 2,9 & 10,51 & 4,0632 & 8943,01 \\
06 & 4,1 & 8,88 & 2,7200 & 5986,67 \\
07 & 4,8 & 17,52 & 3,6664 & 8069,62 \\
\hline
\end{tabular}

Quando comparados os experimentos 01 e 02 quanto ao uso de suspensão ou sobrenadante em torre spray e a fase líquida estando em temperatura ambiente, o experimento 02 , realizado com o sobrenadante, mostrou-se mais eficiente na absorção de $\mathrm{CO}_{2}$. No entanto, deve-se levar em consideração que a concentração de sólidos é muito baixa no sobrenadante, portanto, o processo de absorção de $\mathrm{CO}_{2}$ durante o procedimento experimental se dá basicamente pela reação do $\mathrm{CO}_{2}$ com a $\mathrm{NaOH}$ existente na $\mathrm{LV}$ na fase líquida. Em relação à variação de $\mathrm{pH}$, o experimento 02 apresentou melhores resultados, no entanto foi reduzido o $\mathrm{pH}$ apenas do sobrenadante enquanto que no experimento 01 foi reduzido o $\mathrm{pH}$ da suspensão, com maior teor de sólidos, dessa forma, uma análise mais detalhada é necessária para avaliar a contribuição da quantidade de sólidos na fase líquida para a redução do pH. Ao comparar os experimentos 01 e 03 quanto à variação da temperatura inicial da suspensão a ser usada no processo em torre spray, o experimento 03, realizado a alta temperatura, mostrou-se mais eficiente na absorção de $\mathrm{CO}_{2}$ em relação ao experimento 01, apresentando resultados aproximadamente duas vezes maiores do que os resultados do experimento 01 em relação ao do processo de absorção. A variação do $\mathrm{pH}$ nos dois casos foram bem próximas, no entanto, deve-se levar em consideração que os valores de $\mathrm{pH}$ inicial e final são diferentes nos dois casos, tornando-se semelhante apenas as unidades reduzidas em cada caso.

Quando comparados os experimentos 04 e 06 quanto ao tipo de reator, utilizando sobrenadante com temperatura inicial alta, nota-se que o experimento 06 se mostrou mais eficiente que o experimento 04 na absorção de $\mathrm{CO}_{2}$ para essas condições. No entanto, a variação de $\mathrm{pH}$ nos 
dois casos foi praticamente a mesma. Ao serem comparados os experimentos 05 e 07 quanto ao tipo de reator, utilizando suspensão inicialmente em temperatura ambiente, verifica-se que o experimento realizado na TRE obteve uma maior redução de $\mathrm{pH}$ e maior eficiência quanto à absorção de $\mathrm{CO}_{2}$, pois apesar de os valores de massa absorvida por 100 gramas de LV e massa absorvida anualmente serem maiores no experimento 05, deve-se levar em consideração a quantidade de $\mathrm{CO}_{2}$ que foi absorvida de acordo com a quantidade que entrou no reator, ou seja, a porcentagem de $\mathrm{CO}_{2}$ absorvida foi maior no experimento 07.

\section{CONCLUSÕES}

Ao final das análises dos resultados é possível concluir que os melhores resultados obtidos para redução de $\mathrm{pH}$ e absorção de $\mathrm{CO}_{2}$ ocorreram com a utilização da suspensão de lama vermelha, e dependendo das características do processo ambas as torres são bastante eficientes, no entanto, em relação ao custo de construção e operação, a torre spray é mais vantajosa. Dentre todos os experimentos, o experimento 07 foi o que obteve os melhores resultados, absorvendo $17,52 \%$ de $\mathrm{CO}_{2}$ durante o experimento e reduzindo o $\mathrm{pH}$ da suspensão em 4,8 unidades, quando comparado aos demais em tempo comum a todos. As comparações realizadas entre experimentos 1 e 3 mostram que a variação de $\mathrm{pH}$ não é tão sensível à variação de temperatura quanto a absorção de $\mathrm{CO}_{2}$, visto que as variações no $\mathrm{pH}$ dos experimentos 1 e 3 foram bem próximas, enquanto que a absorção de $\mathrm{CO}_{2}$ para o experimento realizado com temperatura alta foi quase o dobro do valor obtido no experimento realizado a temperatura ambiente. Para uma análise mais detalhada do comportamento da variação do $\mathrm{pH}$ com a variação da temperatura é importante que sejam realizados experimentos na torre de recheio estruturado.

Pode-se concluir, de acordo com os dados coletados, que esse processo é vantajoso e eficiente, visto que foi comprovado que é possível reduzir a alcalinidade da LV, facilitando assim o seu armazenamento e até mesmo a utilização em outras áreas, bem como o sequestro de carbono.

\section{REFERÊNCIAS}

BOBICKE, E. R. et al. Carbon capture and storage using alkaline industrial wastes. Progress in Energy and Combustion Science. v. 38, p. 302-320, 2012.

BONEFANT, D. et al. $\mathrm{CO}_{2}$ sequestration by aqueous red mud carbonation at ambient pressure and temperature. Ind. Eng. Chem. Res. v. 47, p. 7617-7622, 2008.

DILMORE, R. et al. Sequestration of $\mathrm{CO}_{2}$ in mixtures of bauxite residue and saline wastewater. Energy \& Fuels. v. 22, p. 343-353, 2008.

JONES, G. et al. Carbon capture and the aluminium industry-preliminary studies. Environ. Chem. v. 3, p. 297-303, 2006.

SANTOS, I. F. Absorção de gases da queima de combustíveis fósseis em torre de recheio estruturado utilizando lama vermelha. 2013. Dissertação (Mestrado em Engenharia Química) - Universidade Federal do Pará. Belém, 2013.

VENANCIO, L. C. A. Processo de neutralização de resíduo de bauxita com sequestro de carbono. 2010. Dissertação (Mestrado em Engenharia de Materiais) - Instituto Federal de Educação, Ciência e Tecnologia do Maranhão. São Luis, 2010. 